\title{
A randomised double-blind placebo-controlled study on the effects of a herbal toothpaste on gingival bleeding, oral hygiene and microbial variables
}

\author{
S Jayashankar', G J Panagoda', E A P D Amaratunga ${ }^{2}$, K Perera ${ }^{3}$, P S Rajapakse ${ }^{1}$
}

(Index words: herbal toothpaste, oral hygiene, plaque, gingivitis, randomized clinical trial)

\begin{abstract}
Introduction Different systems of traditional medicine of the Indian subcontinent, have used Acacia chundra Willd, Adhatoda vasica Nees., Mimusops elengi L., Piper nigrum L., Pongamia pinnata L. Pirerre, Quercus infectoria Olivier., Syzygium aromaticum L., Terminalia chebula Retz., Zingiber officinale Roscoe., individually or in combinations, to cure oral diseases.

Objective To investigate the oral hygiene and gingival health benefits of toothpaste formulated with a mixture of the above herbs $(15 \% \mathrm{w} / \mathrm{w})$.

Methods Sixty participants (test $n=30$, control $n=30$, mean age $23.6 \pm 2.25$ vs $23.9 \pm 3.2$ years) who fulfilled the selection criteria and had similar plaque $(1.734 \pm 0.29$ vs $1.771 \pm 0.33)$ and percentage of sites with gingival bleeding (19.6 \pm 7 vs $20.7 \pm 8$ ) were studied in a double blind randomised clinical trial. Brushing instructions to all and a scaling for those with calculus were provided two weeks before baseline examination. One $\mathrm{ml}$ of resting saliva was collected to ascertain anaerobic (SAnB) and aerobic (SAB) bacterial counts, plaque index $(\mathrm{PI})$, percentage sites with bleeding on probing (BOP) and pocket depth (PD) (at 6 sites/tooth) were recorded at baseline, followed by home use of the allocated toothpaste (test or placebo) twice a day for 12 weeks. Measurements were repeated at 4,8 , and 12 weeks.
\end{abstract}

Results $\mathrm{PI}, \mathrm{BOP}$ and SAnB decreased significantly in the test group at 4,8 , and 12 weeks compared to baseline measurements (Wilcoxon-Signed Rank Test, $p<0.01$ ). There was no statistically significant improvement in $\mathrm{PI}$, $\mathrm{BOP}$, and SAnB in the placebo group.

Conclusions Our study indicates the beneficial effects of this herbal toothpaste (Sudanth ${ }^{\circledR}$ ) on oral hygiene and gingival health variables when compared with the placebo. Further clinical trials using patients with gingivitis are necessary to confirm the therapeutic benefits of this herbal toothpaste.

Ceylon Medical Journal 2011; 56: 5-9

\section{Introduction}

The use of plant derived medicinal preparations such as poultices, infusions and simple extracts or powders of dried seeds and roots, for treatment of oral diseases, have been used in indigenous systems of medicine since ancient times. Today, traditional medicine is becoming popular probably due to factors such as availability, affordability, cultural familiarity and family influence. Awareness of the public with regard to adverse consequences of over-prescription and misuse of western medicines may also have contributed to this search for solace in traditional or indigenous medicines where herbal preparations constitute a major component [1]. At present the Sri Lankan system of indigenous medicine is a blend of its own 'deshiya chikitsa' system which has been influenced by Ayurvedic system of the Indian subcontinent, the Unani system of Greco-Arabian origin, and the Siddha systems of Dravidian origin $[2,3]$.

Different systems of traditional medicine of the Indian subcontinent, have used Acacia chundra Willd., Adhatoda vasica Nees., Mimusops elengi L., Piper nigrum L., Pongamia pinnata L. Pirerre, Quercus infectoria Olivier., Syzygium aromaticum L., Terminalia chebula Retz., Zingiber officinale Rosce., individually or in combinations to promote oral hygiene and to treat oral diseases due to their beneficial effects on oral health. It has been found that phytochemicals derived from these plants have antibacterial, anti-inflammatory, anticariogenic [4,5] and astringent properties to varying degrees, which can be exploited in the treatment of oral diseases. However, scientific studies or clinical trials investigating the beneficial effects of time-tested indigenous or Ayurvedic medicinal preparations are meagre. The objective of the present study was to assess the effect of a herbal toothpaste $\left(\right.$ Sudantha ${ }^{\circledR}$ ), containing the above herbs on oral hygiene, salivary microbial and gingival health variables.

${ }^{1}$ Department of Oral Medicine and Periodontology, Division of Microbiology, ${ }^{2}$ Department of Oral Pathology, Faculty of Dental Sciences, University of Peradeniya, Sri Lanka, ${ }^{3}$ Department of Engineering Mathematics, Faculty of Engineering, University of Peradeniya, Sri Lanka.

Correspondence: SR, e-mail <sunethraraj@pdn.ac.lk>. Received 30 August and revised version accepted 25 November 2010. Competing interests: We declare that we have no conflicts of interest regarding the work reported here and in the contents of this article. Details of sponsorship of the study are given under 'Acknowledgments'. 


\section{Methods}

The study was a randomised, double-blind, placebocontrolled clinical trial conducted at the Clinical Oral Microbiology Industry Collaborative Research Unit of the Faculty of Dental Sciences, University of Peradeniya, Sri Lanka. Ethical approval for the study was obtained from the Faculty Research and Ethical Review Committee, Faculty of Dental Sciences. Our study was registered with the Sri Lanka Clinical Trials Registry (SLCTR/ 2010 / 002). All participants consented by signing the witnessed agreement voluntarily after receiving verbal and written information about the study.

Study sample consisted of healthy male and nonpregnant female dental students and recent graduates $(n=60)$, aged between 18 and 35 years. Exclusion criteria were as follows: history of herbal toothpaste use within past three months, requirement for prophylactic antibiotic medication, history of antibiotic use within three months, unable to comply with study schedule, untreated caries or periodontitis requiring prompt treatment or current dental treatment, having any medical condition which affects the integrity of the periodontium or oral mucosa, wearing any kind of oral appliance, and history of known allergies.

Two weeks before the baseline examination all 60 participants were given brushing instructions (modified Bass technique [6]) and oral prophylaxis, which included scaling for those with calculus to make all participants free of calculus at the start of the study. They were randomly assigned to the test group $(n=30)$ or the control group $(\mathrm{n}=30)$. At baseline, bleeding on probing (BOP) and probing pocket depth using $15 \mathrm{NC}$ periodontal probes with a ball ended tip (PD) were recorded at 6 sites per tooth-mesio-facial, mid-facial, disto-facial, mesio-lingual, mid-lingual, and disto-lingual. Thereafter a plaque disclosing solution (Dent. Liquid plaque tester, Japan) was applied on the teeth, and the plaque score was recorded at six sites using the Turesky modification [7] of the Quigley and Hein plaque index (PI) [8]. Participants were thereafter given a coded toothpaste (either the herbal or placebo) and advised to brush twice daily for the duration of the 12 week trial, which was completed in July 2010.

Herbal toothpaste and placebo toothpaste were packed in identical tubes and delivered by a person not involved in clinical examination. All investigators and participants were unaware of the identity of the tubes given. Tubes were identified as either the "Group 1" or the "Group 2" tubes. Identity of each tube was revealed only after 12 weeks. No other instructions were given with regard to the use of toothpaste or brushing for the period of the trial. All measurements were repeated at 4, 8, and 12 weeks. In all clinical examinations participants were asked to refrain from brushing for approximately 12 hours before collection of saliva and clinical examination. All examinations were performed by the same examiner.

One $\mathrm{ml}$ of unstimulated saliva was collected and centrifuged at $4000 \mathrm{rpm}$ for 5 minutes, and the pellet obtained was resuspended in $1000 \mu$ l of normal saline and 10-fold dilutions of the resuspended sample were prepared. Then $50 \mu \mathrm{l}$ of $10^{-5}$ and $10^{-6}$ dilutions were separately cultured in 5\% sheep blood agar plates and incubated under aerobic and anaerobic $\left(80 \% \mathrm{~N}_{2}, 10 \% \mathrm{CO}_{2}\right.$ and $\left.10 \% \mathrm{H}_{2}\right)$ conditions in an anaerobic chamber (Te-Her Anaero. Box, Model ANX1(EX), HIRASAWA WORKS, Japan). Total aerobic counts were obtained after 24 hours and total anaerobic counts were obtained after 48 hours, and recorded as total count of bacteria for $1 \mathrm{ml}$ of saliva.

Analysis of data was performed using a statistical package (Minitab for Windows). One participant was the unit of analysis. Plaque score and gingival index scores were averaged on a per participant basis. Each had a whole mouth average score for baseline and another 3 scores for the 4 weeks, 8 weeks and 12 weeks examinations. Likewise each participant had 4 readings each for salivary aerobic and anaerobic counts at baseline, 4 weeks, 8 weeks and 12 weeks. To use the paired T test, differences of pairs should be normally distributed. Anderson-Darling and ShapiroWilk tests were carried out to test for normality $(p<0.05)$ and the differences were not normally distributed. So the equivalent non-parametric test for the paired T test, Wilcoxon-Signed Rank Test was used to analyse the data.

\section{Results}

Sixty participants completed the 12 week trial. One participant was removed from the study because of frothy saliva, which is an indication of reduced salivary secretion, a factor that could affect the salivary microbial counts. The mean age for the test and control groups were $23.6 \pm 2.25$ and $23.9 \pm 3.2$ years respectively. At baseline there were no significant differences $(p>0.05)$ between test and control groups with respect to means of plaque score $(1.73$ vs 1.77), percentage of sites with bleeding on probing (19.6 vs 20.7), pocket depth (2.22 vs $2.22 \mathrm{~mm}$ ), aerobic counts ( 219 vs 214 per $\left.10^{6} \mathrm{ml}\right)$, and anaerobic counts (207 vs 192 per $10^{6} \mathrm{ml}$ ), according to the Mann-Whitney test.

By the end of 12 weeks there was a significant reduction of plaque score, bleeding score and salivary anaerobic bacterial counts in the test group. These findings were statistically significant at 4,8 and 12 weeks when compared with the baseline measurements (Table 1 and Figure 1). In the placebo group measurements of all of the above variables at 4,8 and 12 weeks were not significantly different when compared with that of the baseline measurements (Figure 2 and Table 2). Analysis of the differences between baseline scores and the score at 12 weeks in the placebo and the test group with respect to plaque score, mean bleeding on probing, and salivary aerobic and anaerobic counts showed that the test group had a statistically significant improvement in mean plaque score, bleeding score and total salivary anaerobic counts ( $p<0.001, p<0.001, p<0.013$ respectively) compared to that of the placebo group (Table 3 ). There was no statistically significant change in salivary aerobic counts in both the test and the placebo groups (Table 3). 
Table 1. Placebo-controlled toothpaste trial: plaque score, bleeding score, pocket depth, salivary aerobic and anaerobic bacterial counts in the test group (Group 2)

\begin{tabular}{|c|c|c|c|c|c|}
\hline Variable & Comparisons & $\begin{array}{c}p \text {-value } \\
\text { (two-tailed) }\end{array}$ & $\begin{array}{c}\text { Decision } \\
(\text { alpha }=0.05)\end{array}$ & $\begin{array}{c}p \text {-value } \\
\text { (one-tailed) }\end{array}$ & $\begin{array}{c}\text { Decision } \\
(\text { alpha }=0.05)\end{array}$ \\
\hline \multirow[t]{3}{*}{ PI } & 2nd Vs 1st & 0.003 & Significant & 0.0015 & $1 \mathrm{st}>2 \mathrm{nd}$ \\
\hline & $3 \mathrm{rd} \mathrm{Vs} \mathrm{1st}$ & 0.004 & Significant & 0.0020 & $1 \mathrm{st}>3 \mathrm{rd}$ \\
\hline & 4th Vs 1st & 0.001 & Significant & 0.0005 & $1 \mathrm{st}>4 \mathrm{th}$ \\
\hline \multirow[t]{3}{*}{ PD } & $2 \mathrm{nd} \mathrm{Vs} 1 \mathrm{st}$ & 0.146 & Not significant & & \\
\hline & 3rd Vs 1st & 0.047 & Significant & 0.0235 & $1 \mathrm{st}>3 \mathrm{rd}$ \\
\hline & 4th Vs 1st & 0.073 & Not significant & & \\
\hline \multirow[t]{3}{*}{ BOP } & 2nd Vs 1st & 0.015 & Significant & 0.0075 & $1 \mathrm{st}>2 \mathrm{nd}$ \\
\hline & 3rd Vs 1st & 0.035 & Significant & 0.0175 & $1 \mathrm{st}>3 \mathrm{rd}$ \\
\hline & 4th Vs 1st & 0.004 & Significant & 0.0020 & $1 \mathrm{st}>4$ th \\
\hline \multirow[t]{3}{*}{$\mathrm{SAB}$} & 2nd Vs 1st & 0.039 & Significant & 0.0195 & $1 \mathrm{st}>2 \mathrm{nd}$ \\
\hline & 3rd Vs 1st & 0.130 & Not significant & & \\
\hline & 4th Vs 1st & 0.181 & Not significant & & \\
\hline \multirow[t]{3}{*}{ SAnB } & 2nd Vs 1st & 0.030 & Significant & 0.015 & $1 \mathrm{st}>2 \mathrm{nd}$ \\
\hline & 3rd Vs 1st & 0.014 & Significant & 0.007 & $1 \mathrm{st}>3 \mathrm{rd}$ \\
\hline & 4th Vs 1st & 0.003 & Significant & 0.0015 & $1 \mathrm{st}>4$ th \\
\hline
\end{tabular}

$\mathrm{PI}=$ Plaque index $\mathrm{PD}=$ Pocket depth $\mathrm{BOP}=$ Bleeding on probing $\mathrm{SAB}=$ Aerobic bacterial counts in saliva $\mathrm{SAnB}=$ Anaerobic bacterial counts in saliva

Table 2. Placebo-controlled toothpaste trial: plaque score, bleeding score, pocket depth, salivary aerobic and anaerobic bacterial counts in the control group (Group 1)

\begin{tabular}{|c|c|c|c|}
\hline Variable & Comparisons & $\begin{array}{l}p \text {-value } \\
(2 \text {-tailed })\end{array}$ & $\begin{array}{c}\text { Decision } \\
(\text { alpha }=0.05)\end{array}$ \\
\hline PI & $\begin{array}{l}\text { 2nd Vs 1st } \\
\text { 3rd Vs 1st } \\
\text { 4th Vs 1st }\end{array}$ & $\begin{array}{l}0.976 \\
0.991 \\
0.113\end{array}$ & $\begin{array}{l}\text { Not significant } \\
\text { Not significant } \\
\text { Not significant }\end{array}$ \\
\hline PD & $\begin{array}{l}\text { 2nd Vs 1st } \\
\text { 3rd Vs 1st } \\
\text { 4th Vs 1st }\end{array}$ & $\begin{array}{l}0.469 \\
0.984 \\
0.723\end{array}$ & $\begin{array}{l}\text { Not significant } \\
\text { Not significant } \\
\text { Not significant }\end{array}$ \\
\hline BOP & $\begin{array}{l}\text { 2nd Vs 1st } \\
\text { 3rd Vs 1st } \\
\text { 4th Vs 1st }\end{array}$ & $\begin{array}{l}0.990 \\
0.623 \\
0.151\end{array}$ & $\begin{array}{l}\text { Not significant } \\
\text { Not significant } \\
\text { Not significant }\end{array}$ \\
\hline SAB & $\begin{array}{l}\text { 2nd Vs 1st } \\
\text { 3rd Vs 1st } \\
\text { 4th Vs 1st }\end{array}$ & $\begin{array}{l}0.943 \\
0.696 \\
0.245\end{array}$ & $\begin{array}{l}\text { Not significant } \\
\text { Not significant } \\
\text { Not significant }\end{array}$ \\
\hline SANB & $\begin{array}{l}\text { 2nd Vs 1st } \\
\text { 3rd Vs 1st } \\
\text { 4th Vs 1st }\end{array}$ & $\begin{array}{l}0.959 \\
0.181 \\
0.581\end{array}$ & $\begin{array}{l}\text { Not significant } \\
\text { Not significant } \\
\text { Not significant }\end{array}$ \\
\hline
\end{tabular}

$\mathrm{PI}=$ Plaque index $\quad($ etc - as above $)$ 
Table 3. Analysis of the differences between baseline scores and the score at 12 weeks in the control and the test groups with respect to plaque score, mean bleeding on probing and salivary aerobic and anaerobic counts

\begin{tabular}{lcl}
\hline Control vs Test & p-value & Conclusion \\
\hline Mean plaque index & $<0.001$ & Control $<$ Test 2 \\
Mean pocket depth & $=0.211$ & No significant difference \\
Mean bleeding on probing & $<0.001$ & Control $<$ Test 2 \\
Mean aerobic counts x $10^{6}$ & 0.185 & No significant difference \\
Mean anaerobic counts x $10^{6}$ & $<0.013$ & Control $<$ Test 2 \\
\hline
\end{tabular}

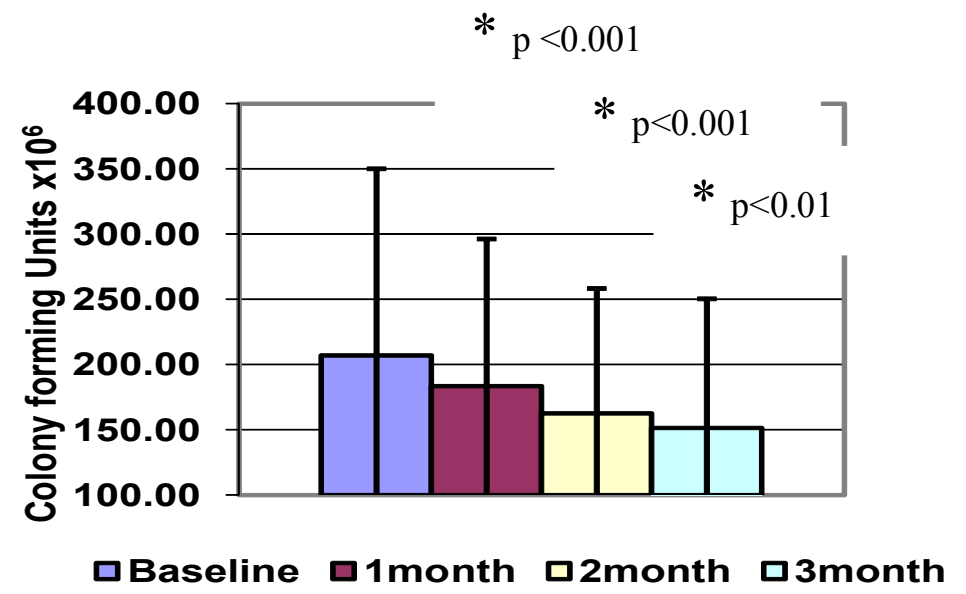

Figure 1. Mean anaerobic counts in $1 \mathrm{ml}$ of saliva in the test group.

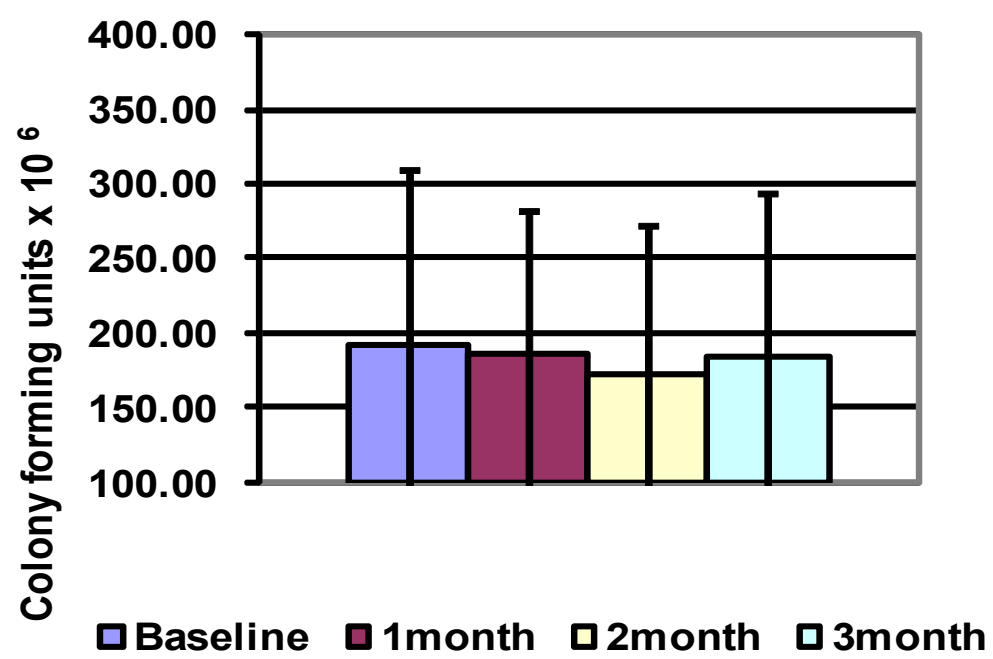

Figure 2. Mean anaerobic counts in $1 \mathrm{ml}$ of saliva in the placebo group. 


\section{Discussion}

In the present study, a toothpaste containing the herbal ingredients dried heartwood of Acacia chundra Willd., dried leaves of Adhatoda vasica Nees., dried bark of Mimusops elengi L., dried seeds of Piper nigrum L., dried roots of Pongamia pinnata L. Pirerre, dried gall of Quercus infectoria Olivier., dried flower bud of Syzygium aromaticum L., dried fruit of Terminalia chebula Retz., dried rhizome of Zingiber officinale Rosce., was tested for its efficacy during 12 weeks of twice daily use in improving gingival and oral hygiene and salivary microbial variables. In indigenous systems of medicine, different components of these plants have been used in medicinal preparations to clean teeth or to treat oral diseases such as pyorrhea and periodontal diseases $[1,2,3]$.

Anti-inflammatory actions of some of the ingredients such as Pongamia pinnata and Zingiber officinale Roscoe have been reported [10]. Quercus infectoria Oliv., and Terminalia chebula - aralu, are also known to have antibacterial activities [11]. Potent growth inhibitory effects of Syzygium aromaticum (Clove) have been shown against Gram negative, anaerobic periodontal pathogens such as $P$. gingivalis and P. intermedia [12]. Further, leaf extracts of Adhatoda vasica Nees., have been shown to improve gingival inflammation with resultant reduction in bleeding [4]. Mimusops elengi L ('Munnamal') has long been used in indigenous systems of medicine as a specific cure for diseases of the gums and teeth, as well as other diseases affecting mankind [13].

The findings of the present study showed statistically significant reductions in all variables tested (gingival bleeding, oral hygiene and salivary anaerobic bacterial counts) except salivary aerobic bacterial counts. The placebo-controlled group did not show significant reductions in any of the variables. Hence this study provides evidence for the beneficial effects of the test herbal toothpaste on gingival bleeding, plaque levels and the salivary anaerobic counts.

Since none of the study groups were given any special brushing instructions or professional interventions after the screening visit, it is likely that the improvements in number of sites with gingival bleeding, plaque score and salivary anaerobic counts in the test group are due to the herbal ingredients in the test toothpaste. Especially significant is the reduction in anaerobic bacterial counts which may explain the improvements observed in gingival bleeding. Reduction in bleeding could also be attributed to anti-inflammatory properties of the herbal ingredients. Further studies are necessary to clarify the activity of the individual herbs included in this toothpaste and the activity of different combinations of the herbs in the test herbal toothpaste.

Since our results show that the test herbal toothpaste $\left(\right.$ Sudantha $\left.{ }^{\circledR}\right)$ is effective in significantly improving the variables we have examined, it would be appropriate to test it against other toothpastes available in the market, using the same criteria. Clinical trials including patients with established gingivitis are indicated to investigate the therapeutic use of this herbal toothpaste.

\section{Acknowledgements}

We are thankful to staff of the Veterinary Research Institute, Gannoruwa for providing sheep blood for this project, and Mrs. MRDM Senanayake of the Division of Microbiology, Faculty of Dental Sciences for technical assistance. Link Natural Products (Pvt) Ltd., provided funding for the entire study.

\section{References}

1. Cowan MM. Plant products as antimicrobial agents. Clinical Microbiology Reviews 1999; 12: 564-82.

2. Nanayakkara V, Ekanayake SL. Use of traditional medicine for oral conditions in rural Sri Lanka. International Dental Journal 2008; 58: 86-90.

3. Pilapitiya U. Traditional medicine. Regional Health Forum 1996; 1: 51.

4. Doshi JJ, Patel VK, Venkatakrishna B. Effect of Adhatoda vasica massage in pyorrhea. International Journal of Crude Drug Research 1983; 4: 173-6.

5. Modder WW, Amarakoon AMT. Tea and Health. Tea Research Institute of Sri Lanka. 2002.

6. Bass CC. An effective method of personal hygiene. Journal of Louisiana Medical Society 1954: 106: 100-8.

7. Turesky S, Gilmore ND, Glickman I. Reduced plaque formation by the chloromethyl analogue of vitamin C. Journal of Periodontology 1970; 41: 41-3.

8. Quigley G, Hein J. Comparative cleaning efficiency of manual and power brushing. Journal of the American Dental Association 1962; 65: 26-9.

9. Jindal MN, Patel VK. Textbook of General and Dental Pharmacology. B. S. Shah Publishers, Ahmedabad, India 1978.

10. Chopade VV, Tankar AN, Pande VV, et al. Pongamia pinnata, phytochemical constituents traditional uses and pharmacological properties: a review. International Journal of Green Pharmacy 2008; 2: 72-5.

11. Carounanidy U, Satyanarayanan R, Velmurugan A. Use of an aqueous extract of Terminalia chebula as an anticaries agent: a clinical study. Indian Journal of Dental Research 2007; 18: 152-6.

12. Lining C, Christine DW. Compounds from Syzygium aromaticum possessing growth inhibitory activity against oral pathogens. Journal of Natural Products 1996; 59: 987-90.

13. Mitra R. Bakula - a reputed drug of Ayurveda, its history and uses in Indian medicine. Indian Journal of History of Science 1981; 16: 169-80. 